\title{
Galaxies in 3D across the Universe
}

\author{
Denis Burgarella ${ }^{1}$, Toru Yamada ${ }^{2}$, Giovanni Fazio ${ }^{3}$ and \\ Marcin Sawicki ${ }^{4}$ \\ ${ }^{1}$ Aix-Marseille Universit, CNRS, LAM (Laboratoire d'Astrophysique de Marseille) UMR 7326, \\ 13388, Marseille, France \\ email: denis.burgarella@lam.fr \\ ${ }^{2}$ Astronomical Institute, Tohoku Univ., Japan \\ email: yamada@astr.tohoku.ac.jp ${ }^{3}$ Harvard Smithsonian Center for Astrophysics, Cambridge \\ Massachussets, USA \\ email: gfazio@cfa.harvard.edu ${ }^{4}$ Department of Astronomy and Physics and Institute for \\ Computational Astrophysics, Saint Mary's University, Halifax, Nova Scotia, Canada \\ email: sawicki@ap.smu.ca and the WISH team
}

\begin{abstract}
WISH is a new space science mission concept whose primary goal is to study the first galaxies in the early universe. The primary science goal of the WISH mission is to push the highredshift frontier beyond the epoch of reionization by utilizing its unique imaging and spectrocopic capabilities and the dedicated survey strategy. WISH will be a $1.5 \mathrm{~m}$ telescope equipped with a $1000 \operatorname{arcmin}^{2}$ wide-field Near-IR camera to conduct unique ultra-deep and wide-area sky imaging surveys in the wavelength range $1-5 \mu \mathrm{m}$. A spectroscopic mode (Integral-Field Unit) in the same Near-IR range and with a field of view of $0.5-1$ arcmin and a spectral resolution $\mathrm{R}=$ 1000 is also planned. The difference between WISH and EUCLID in terms of wavelength range explains why the former concentrates on the reionization period while the latter focuses on the universe at $z<3$. WISH and JWST feature different instantaneous fields of view and are therefore also very complementary.
\end{abstract}

Keywords. universe: first light - reionization - galaxies: evolution - galaxies: formation - space telescope - near-infrared

\section{Where should we statistically search and study the first galaxies?}

WISH is designed to detect the first star-forming galaxies in the universe and to follow them up in the early phases of their evolution. One of the first questions that we need to address is which wavelength range would be the best to reach the above objective: the rest-frame far-ultraviolet (FUV) in the rest-frame far-infrared (FIR)? In a recent paper, Burgarella et al. (2013) showed that the FUV dust attenuation $\left(\mathrm{A}_{F U V}\right)$ reaches the same level at $z=3.6$ than at $\mathrm{z}=0$ (Fig. 1). This suggests that the early universe would be better studied in the rest-frame FUV. At $z>5$, this FUV range moves into the near-infrared $(1-5 \mu \mathrm{m})$, in the WISH spectral range. Of course, it does not mean that dusty galaxies do not exist at high redshifts as shown by the recent discoveries of, e.g., FLS3 (Riechers et al.2013) at $z=6.34$. The above statement should be accepted as a statistical one unless we misunderstand the early star formation history of the universe.

\section{What is WISH?}

WISH will adopt an optimized strategy to build the photometric surveys and finally reach the planned depth (Fig. 2). This strategy will make the best use of the individual images to detect transient objects like, e.g., type Ia Supernova. A unique optical layout (Fig. 3) has been developed to achieve the diffraction-limited imaging at $1-5 \mu \mathrm{m}$ over the 


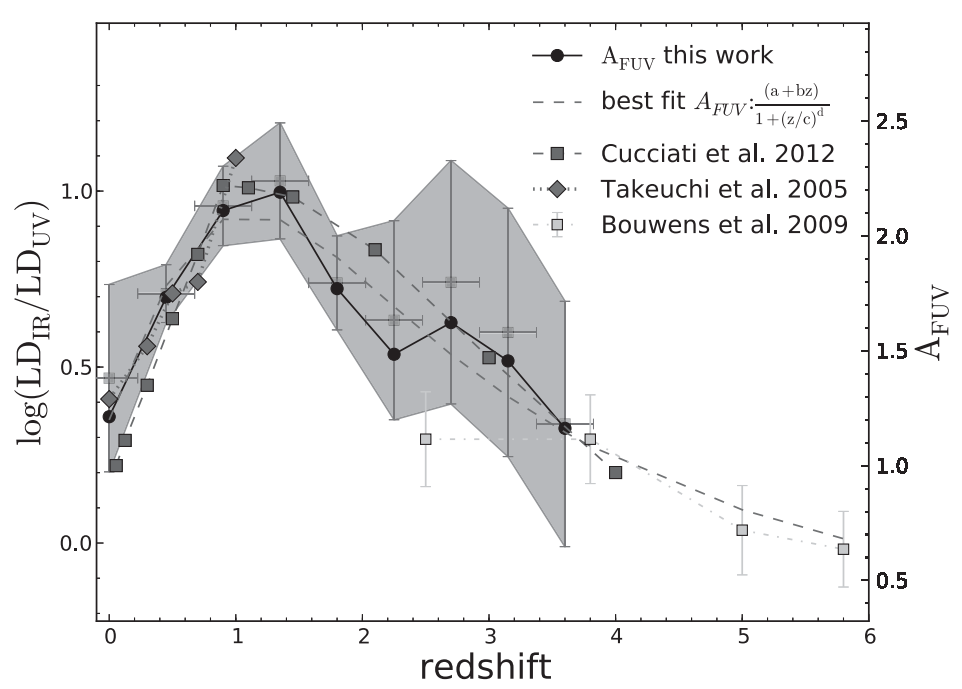

Figure 1. Left axis: The ratio of the FIR-to-FUV luminosity density $(I R X)$ is shown here. The right axis indicates the FUV dust attenuation $\left(\mathrm{A}_{F U V}\right)$ after converting the FIR-to-FUV ratio using Burgarella et al. (2005). The red dotted line with red diamonds is taken from Takeuchi, Buat \& Burgarella (2005). The green filled area and green dots are the associated uncertainties estimated through bootstrapping. Black dots denote the values directly computed from the luminosity functions. At $z=3.6, \mathrm{~A}_{F U V}$ reaches about the same value as at $z=0$. Takeuchi et al. (2005) (red diamonds) used an approach identical to ours while a SED analysis (no FIR data) is performed in Cucciati et al. (2012) (blue boxes). Bouwens et al. (2009) are estimates based on the UV slope $\beta$.

\begin{tabular}{|l|l|l|l|}
\hline & $\begin{array}{l}\text { Depth }(\mathbf{3} \boldsymbol{\sigma}) \\
(\mathbf{A B ~ m a g})\end{array}$ & Area & $\begin{array}{l}\text { Example of the Filters } \\
\text { (a plan, to be determined) }\end{array}$ \\
\hline Ultra Deep Survey & $\mathbf{2 8}$ & $\mathbf{1 0 0 \mathrm { deg } ^ { 2 }}$ & $\mathbf{1 . 4 , 1 . 8 , 2 . 3 , 3 . 0}$ micron \\
\hline Multi-Band Survey & $\mathbf{2 8}$ & $\mathbf{1 0} \mathrm{deg}^{2}$ & $\mathbf{1 . 0 , 4 . 0}$ \\
\hline Ultra Wide Survey & $24-25$ & $1000 \mathrm{deg}^{2}$ & $1.4,1.8,2.3$ \\
\hline Extreme Survey & $29-30$ & $0.25 \mathrm{deg}^{2}$ & $1.0,1.4,1.8$ \\
\hline
\end{tabular}

Figure 2. WISH strategy with the different surveys designed tooptimize the photometric detection of $z>10$ galaxies.

required large area with an angular resolution of about $0.2 \operatorname{arcsec}$ FWHM at $1.5 \mu \mathrm{m}$. The $1.5 \mathrm{~m}$ primary mirror will be cooled by passive cooling in the stable thermal environment near the Sun-Earth L2 at about 100K to achieve the zodiacal light limited imaging.

\section{An IFU spectrograph for WISH}

The spectroscopic mode is studied in France under the responsability of the Laboratoire d'Astrophysique de Marseille, France. The baseline is a 1x1 $\operatorname{arcmin}^{2}$ Integral-Field Unit (IFU) using 40 (TBC) slicers already studied for the SNAP project. The design shows that the spectrograph could observe in a parallel mode (Fig. 3) which translates into spectroscopic exposure times as long as the telescope lifetime (within uncertainties due to the overheads).

WISH-Spec will allow to acquire very large samples of high-redshift and primordial galaxies by combining spectroscopic detections within total (non contiguous) 1- $\mathrm{deg}^{2}$ and 10- $\mathrm{deg}^{2}$ fields of views (Fig. 4). Therefore, the first strong advantage of WISH-Spec wrt JWST is that $10^{4}-10^{5}$ faint emission-line galaxies in $1 \mathrm{deg}^{2}$ (Fig. 5 left) and $10^{3}-10^{6}$ 


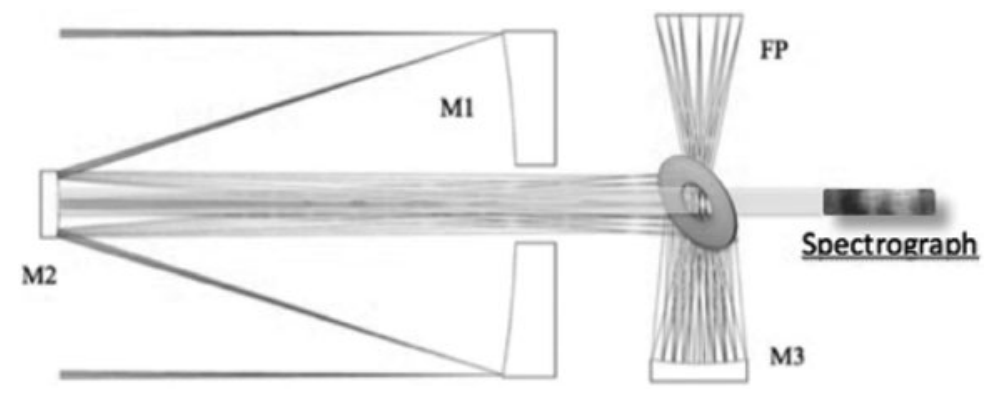

Figure 3. WISH optical design. Light will go to the spectrograph WISH-Spec through a hole in a flat mirror. WISH-Spec could therefore observe in a parallel mode and make the best use of the telescope lifetime to build wide and deep spectroscopic surveys over 10 and $1 \mathrm{deg}^{2}$, respectively.

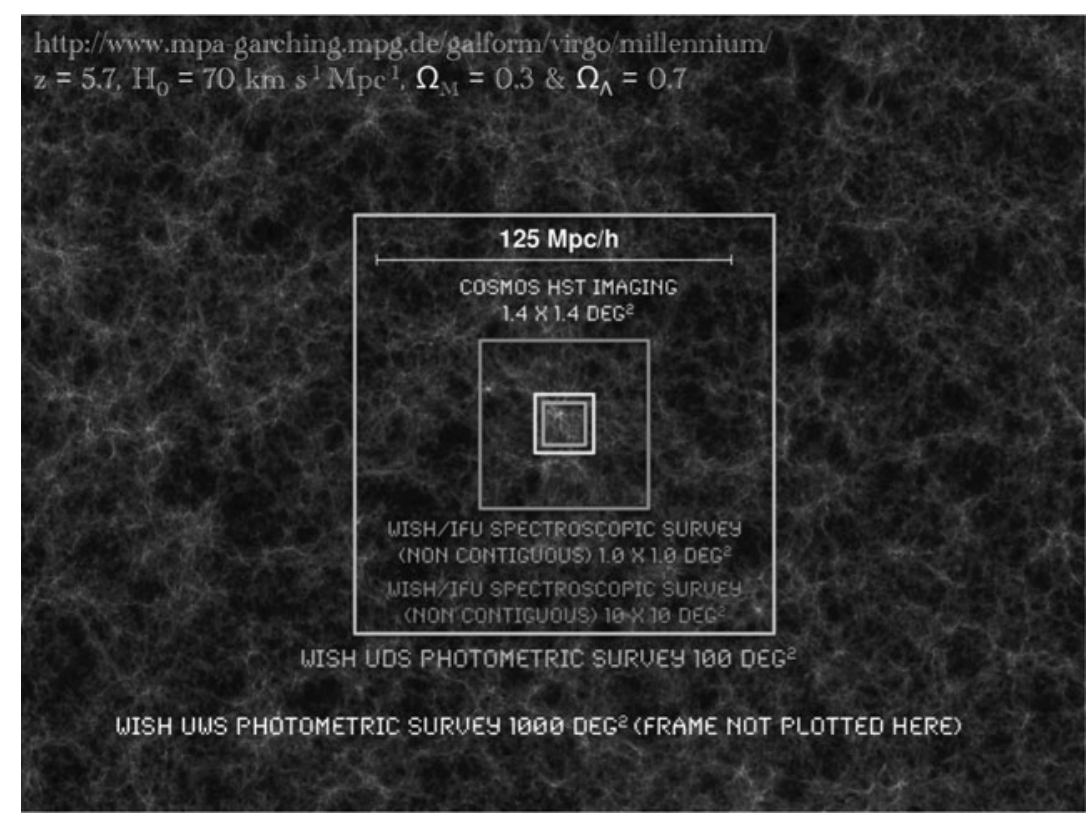

Figure 4. WISH spectroscopic surveys

bright emission-line galaxies in $10 \mathrm{deg}^{2}$ in the redshift range $3<z<8$ (Fig. 5 right) will be detected.

\section{Conclusions}

It is important to stress that WISH will be complementary to EUCLID since the former will concentrate on the reionization times at $z>6$ in the wavelength range $1-5 \mu \mathrm{m}$ while the latter will focus on lower redshifts $(z<2-3)$ by construction since EUCLID's wavelength range is limited to $\sim 2 \mu \mathrm{m}$, and is not optimized to study the first galaxies.

WISH will also be complementary to JWST. The wavelength ranges are the same but the field of view is much larger for WISH both photometrically (900 $\operatorname{arcmin}^{2}$ for WISH and $9 \operatorname{arcmin}^{2}$ for NIRCam) and spectroscopically (even though JWST will feature a MOS over $3 \times 4 \operatorname{arcmin}^{2}$, JWST's IFU will only cover $3 \times 3 \operatorname{arcsec}^{2}$ while WISH's IFU will be a factor of 10 larger to $0.5-1$ arcmin). 

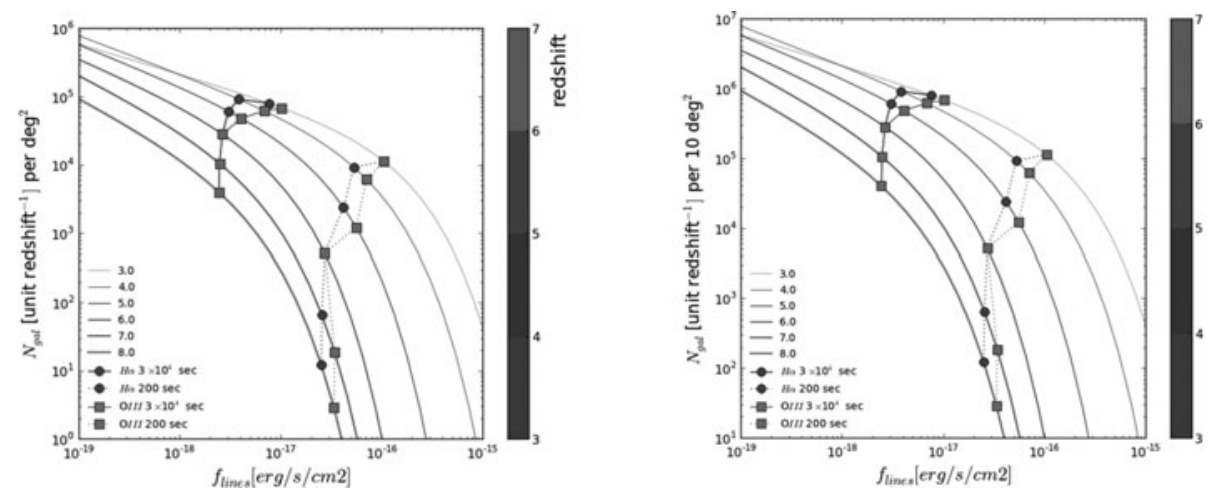

Figure 5. Expected number of spectroscopically detected galaxies at $3<z<10$ estimated by translating Far-UV luminosity functions into $\mathrm{H} \alpha$ luminosity function for the $1 \mathrm{deg}^{2}$ Deep Spectroscopic Survey (left) and the $10 \mathrm{deg}^{2}$ Wide Spectroscopic Survey (right).

At very high redshifts, WISH photometric surveys will provide $10^{3}-10^{4}$ galaxies at $z \sim 12$ and $10^{2}$ at $z \sim 15$ (depending on assumed evolution) at $\mathrm{AB} \sim 27-28$ from the $100-\mathrm{deg}^{2}$ UDS survey. Following up these galaxies spectroscopically requires ELTs. The E-ELT limiting magnitudes in spectroscopy reach about $\mathrm{AB}=27-28$ for the longest exposure times. This means that fainter (e.g., JWST) objects might be difficult to follow up even with ELTs while WISH ones would be more easily observed with the E-ELT spectrographs.

In the range $3<z<8$ WISH-Spec will also build large samples of galaxies over non contiguous fields of $1 \mathrm{deg}^{2}$ and $10 \mathrm{deg}^{2}$ observed spectroscopically with $\mathrm{R}=1000$. We will be able to study the physics of the galaxies and their very early phases of formation around the epoch of reionization.

\section{Acknowledgements}

A support from the French Centre National d'Etudes Spatiales (CNES), the Programme National Cosmologie et Galaxies and the LABEX OCEVU (Origines, Constituants \& EVolution de lUnivers) is acknowledged.

\section{References}

Bouwens, R J., Illingworth, G. D., Franx, M., et al. 2009, ApJ 705, 936

Burgarella D., et al., 2013, A\& A 554, 70

Burgarella, D., Buat, V., \& Iglesias-P/'aramo, J., 2005, MNRAS 360, 1413

Cucciati, O., Tresse, L., Ilbert, O., et al. 2012, A\&A 539, 31

Riechers, D., et al. 2013, Nature 496, 329

Takeuchi, T. T., Buat, V., \& Burgarella, D., 2005, A\&A 440, L17 